\title{
Propuesta Metodológica de Análisis para la Inserción de Futuros Nodos Comerciales en la Ciudad de Manizales (Colombia)
}

\author{
Diego A. Escobar(1), Jorge A. Montoya ${ }^{(1)}$ y Carlos A. Moncada ${ }^{(2)}$ \\ (1) Facultad de Ingeniería y Arquitectura, Departamento de Ingeniería Civil, Universidad Nacional de \\ Colombia - Sede Manizales. (e-mail: daescobarga@unal.edu.co; joamontoyago@unal.edu.co) \\ (2) Facultad de Ingeniería, Departamento de Ingeniería Civil y Agrícola. Universidad Nacional de Colombia \\ - Sede Bogotá. (e-mail: camoncadaa@unal.edu.co)
}

Recibido Jul. 17, 2017; Aceptado Sep. 28, 2017; Versión final Oct. 5, 2017, Publicado Abr. 2018

\begin{abstract}
Resumen
Se muestran los resultados del análisis comparativo de la accesibilidad territorial entre los nodos comerciales actuales y los que se encuentran propuestos para su construcción en la Ciudad de Manizales (Colombia). El estudio tiene como objetivo determinar el nivel de cobertura poblacional e identificar los sectores más beneficiados con la ubicación de los nuevos nodos comerciales. En la actualidad, la posibilidad de acceder a nodos de comercio, servicios y recreación, juega un papel importante en los procesos de desarrollo y planificación de una ciudad. Dichos usos se agrupan, en ocasiones, en puntos estratégicos de la ciudad identificados como nodos o centros comerciales. La ubicación de estos nodos comerciales debe ser tal que abarque la mayor cantidad de población posible en los menores tiempos medios de viaje para los usuarios. Los resultados muestran que son los sectores periféricos del área de estudio las que mayor beneficio reportan, coincidiendo con ser las áreas que mayor deficiencia de accesibilidad presentan actualmente.
\end{abstract}

Palabras clave: accesibilidad; planificación de la ciudad; localización del comercio; tiempo medio de viaje

\section{Methodological analysis proposal for the insertion of future commercial nodes in the city of Manizales (Colombia)}

\begin{abstract}
In this research, a comparative analysis of the territorial accessibility between the current commercial nodes and those proposed for its construction in the city of Manizales (Colombia) is done. The objective of the study was determining the level of population coverage and identifying the sectors most benefited with the location of the new commercial nodes. Currently, the possibility of accessing to the nodes of commerce, services and recreation areas, plays an important role in the development and planning of a city. These land uses are sometimes grouped in strategic points identified as nodes or shopping malls. The location of these commercial nodes should be selected so they cover a large population using the smallest possible travel time for users. The results show that are the peripheral sectors of the areas under study those showing the greatest benefit, coinciding with being the areas that nowadays present the greatest deficiency of accessibility.
\end{abstract}

Keywords: accessibility; city planning; commercial centers location; average travel time 


\section{INTRODUCCIÓN}

Manizales, capital del departamento de Caldas, se encuentra localizada en la región centro - occidente de Colombia (Fig. 1) sobre la cordillera andina a una altitud media de 2150 m.s.n.m; cuenta con una extensión total de $572 \mathrm{~km}^{2}$ (Gobernación de Caldas, 2016). El área de estudio abarca la conurbación Manizales Villamaría, cuya topografía presenta elevaciones fuertes, generando limitaciones y dificultades tanto en los procesos de urbanización (Robledo, 1996) como de inserción de nuevas infraestructuras; situación que se acentúa en la periferia del área de estudio, ya que se identifican desarrollos informales en altas pendientes, conllevando a que existan deficiencias en la prestación de servicios (Chardon, 2008; Torres, 2009). El área de estudio asciende a $58,7 \mathrm{~km}^{2}$, en la que se asienta una población de 415.124 habitantes, de los cuales 369.981 (89\%) pertenecen a Manizales (CIE, 2016) y el resto pertenece al municipio contiguo de Villamaría.
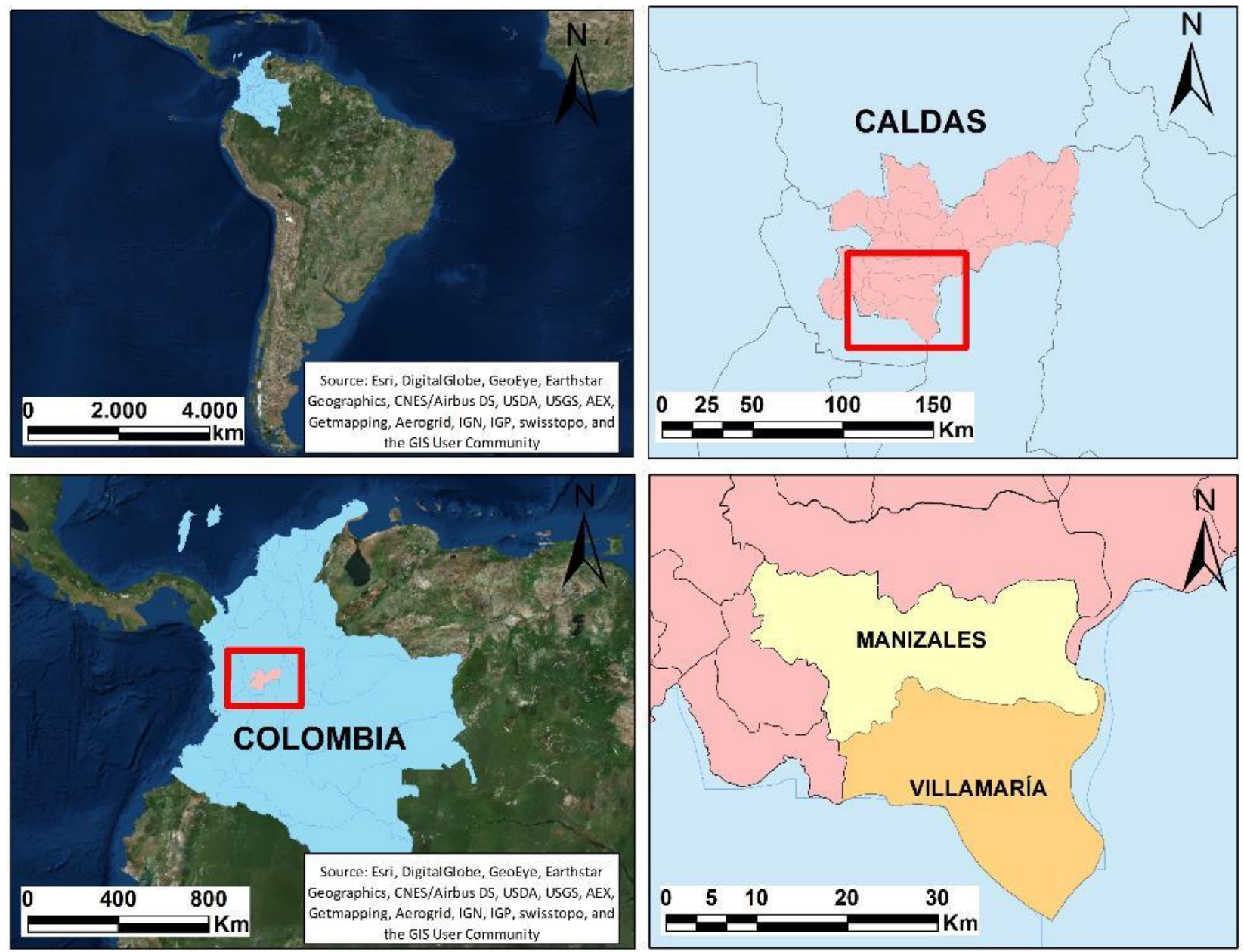

Fig. 1: Localización geográfica de la ciudad de Manizales y Villamaría.

La planificación y desarrollo de una ciudad se debe soportar en adecuadas estrategias que promuevan, en conjunto, distribución espacial de actividades y políticas de gestión del transporte (Hasibuna et al., 2014), articulando directamente dichas estrategias con aspectos de desarrollo sostenible como usos del suelo, estructura urbana y movilidad. Es de resaltar que una adecuada distribución geoespacial de usos del suelo y actividades, permite que se presenten patrones de viajes (Escobar et al., 2013), en unas ocasiones recurrentes, lo cuales dependen no sólo de las distancias a recorrer, sino del modo de transporte usado para realizar el desplazamiento.

En relación con las características de movilidad en la ciudad de Manizales, a pesar que el transporte público colectivo aún reporta valores porcentuales de viajes (45\%) superiores al del conjunto moto y vehículo particular (32\%), el primero ha dejado de movilizar un $5 \%$ del total de pasajeros en el periodo $2014-2015$, mientras que el parque automotor se incrementó en un $9 \%$ (MCV, 2016). Actualmente, la carga vehicular se encuentra soportada en la red de infraestructura del transporte de la ciudad, la cual posee una extensión total de $749 \mathrm{~km}$ (Alcaldía de Manizales, 2011) y ha sido intervenida constantemente con el fin de satisfacer las necesidades de movilidad. Esta situación se observa con bastante preocupación en horas pico relacionadas con el acceso a infraestructuras de comercio como los centros comerciales, los cuales, en horarios bastante puntuales, atraen una importante cantidad de personas y vehículos, generando problemas de movilidad en 
las vías cercanas. Lo anterior tiene una relación directa con las características de accesibilidad que la red de infraestructuras ofrezca a ciertos sectores de la ciudad, como en este caso de estudio, los centros comerciales; de aquí que el termino accesibilidad se encuentre estrechamente relacionado con el desarrollo del uso del suelo (Hansen, 1959).

El concepto de "accesibilidad" posee numerosas y variadas definiciones, dentro de las cuales encontramos algunas tan simples como la facilidad con la cual las personas pueden acceder a oportunidades o servicios (Wachs y Kumagai, 1973) o tan compactas como la intensidad de posibilidades para el intercambio (Engwicth, 1993), sin embargo, la primer interpretación técnica de accesibilidad, fue expresada por Hansen (1959) como "The potencial of opportunities for interaction", aunque el uso del concepto se remonta hasta la segunda década de siglo XX (Batty, 2009). Algunas interpretaciones más externas, plantean la accesibilidad en dos clases, potencial y real (Garrocho y Campos, 2006), las cuales se relacionan con la probabilidad de acercamiento e interacción (Martínez, 2012) y el uso comprobable del servicio, respectivamente. Buscado una definición más profunda y acorde al desarrollo de esta investigación, se establece la accesibilidad como la relación entre formas de actividad humana, considerando la movilidad, comprensión y comunicación (Morris et al, 1979); siendo sujetas a limitaciones de alcance dada la existencia de barreras (Geurs y Van Wee, 2004; Vega, 2011). Las principales variables a considerar en un análisis de accesibilidad territorial son los nodos de actividad, modos de transporte, velocidad y distancia; con estas, es factible desarrollar numerosos tipos de investigación dentro de los cuales se abordan temas como: cohesión social (Schürmann et al, 1997); marketing (Geurs y van Eck, 2001), prestación y localización de servicios (Calcuttawala, 2006), exclusión social (Bocarejo y Oviedo, 2012; Preston y Rajé, 2007); sostenibilidad (Escobar et al., 2015a), operatividad del transporte (Escobar et al., 2015b); entre otros.

Así pues, conociendo sobre la necesidad de proveer unas adecuadas condiciones de accesibilidad territorial hacia centros de comercio, servicios y recreación como componente fundamental para la planificación, crecimiento y desarrollo de una ciudad, mediante la presente investigación se propone una metodología de análisis para el cálculo del impacto que sobre la red de infraestructuras y sobre la distribución poblacional produciría la inserción de establecimientos multifuncionales en los cuales se permita agrupar cada tipo de servicio, minimizando el tiempo de búsqueda y aproximación; sin desconocer la existencia de profundos desarrollos metodológicos que abordan el análisis locacional de unidades comerciales en función de variables teóricas, técnicas de jerarquización y modelos de interacción espacial (Garrocho et al., 2002). Luego de la introducción se presenta la metodología de investigación, posteriormente se muestran los resultados obtenidos y discusión de los mismos, para finalizar con las principales conclusiones obtenidas.

\section{METODOLOGÍA}

La metodología empleada en esta investigación se compone de 4 fases principales que se muestran en la Fig. 2: 1) Consecución, actualización y puesta a punto de la red de infraestructura vial; 2) Georreferenciación de los puntos de estudio (Nodos o Centros Comerciales); 3) Análisis de accesibilidad territorial; 4) Análisis de cobertura.

Etapa 1, Consecución, actualización y puesta a punto de la red de infraestructura vial. Inicialmente se procede a analizar, intervenir y verificar el funcionamiento de la red de infraestructura vial para la ciudad de Manizales (Fig. 3), obtenida del Plan de Movilidad (Alcaldía de Manizales, 2011) y compuesta por la interacción básica de 2 tipos de elementos, nodos y arcos, los cuales representan las intersecciones y segmentos viales respectivamente; en este proceso, es de vital importancia analizar la velocidad de operación de cada arco, ya que esta se convierte en un elemento principal para el cálculo de Accesibilidad territorial (Geurs y van Eck, 2001). La estructuración es llevada a cabo mediante el uso de la herramienta ArcMap, en la cual se consideraron las intervenciones realizadas en el periodo 2010 - 2017 (construcciones viales, cambios direccionales, etc.).

Etapa 2, Georreferenciación de los puntos de estudio (Nodos o Centros Comerciales). Los puntos analizados en esta investigación son un total de ocho, de los cuales cinco son nodos o centros comerciales existentes (P1 Cable Plaza, P2 Fundadores, P3 Parque Caldas, P4 Multicentro y P5 Sancancio), los cuales fueron estudiados de forma conjunta e independiente por Zuluaga y Escobar (2017); y tres son nodos o centros comerciales cuya localización se fundamentan en un análisis urbanístico preliminar, definiendo que dos se encuentran en etapa de propuesta (P6 La Enea y P7 Chipre) y uno en etapa de construcción (P8 Mall Plaza). En la Fig. 3 se observa la georreferenciación de los puntos de estudio.

Etapa 3, Análisis de accesibilidad territorial. Posterior a la localización de los puntos de estudio, se establecen las características necesarias para la construcción de las curvas de accesibilidad territorial en ArcMap, en la cual los centros comerciales adquieren la denominación de "Facilidades", la intersección entre arcos se identifica como "Incidentes" y la configuración o desplazamiento entre arcos como "Rutas”, terminología propia de ArcMap. 


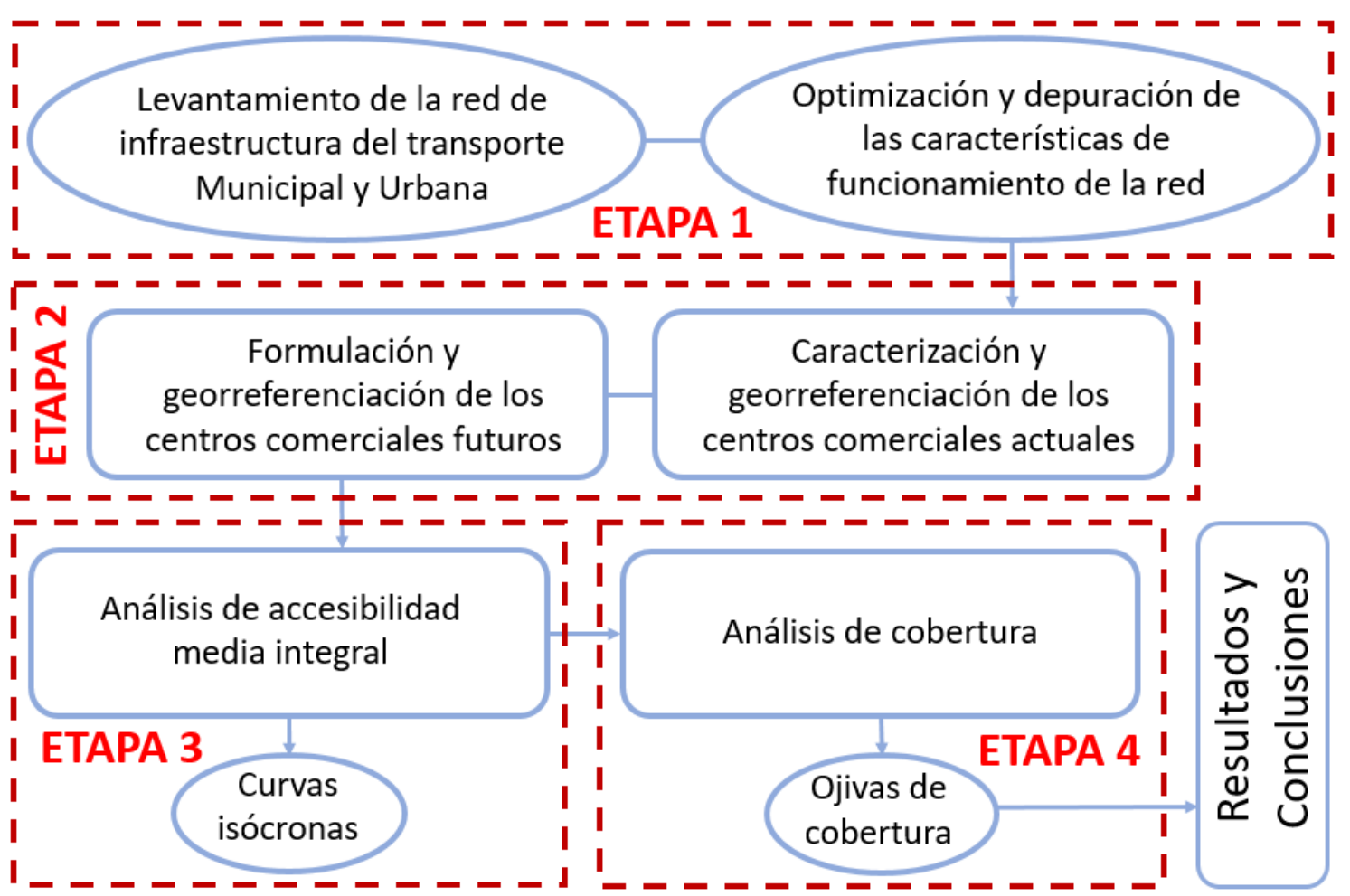

Fig. 2: Metodología de la investigación.

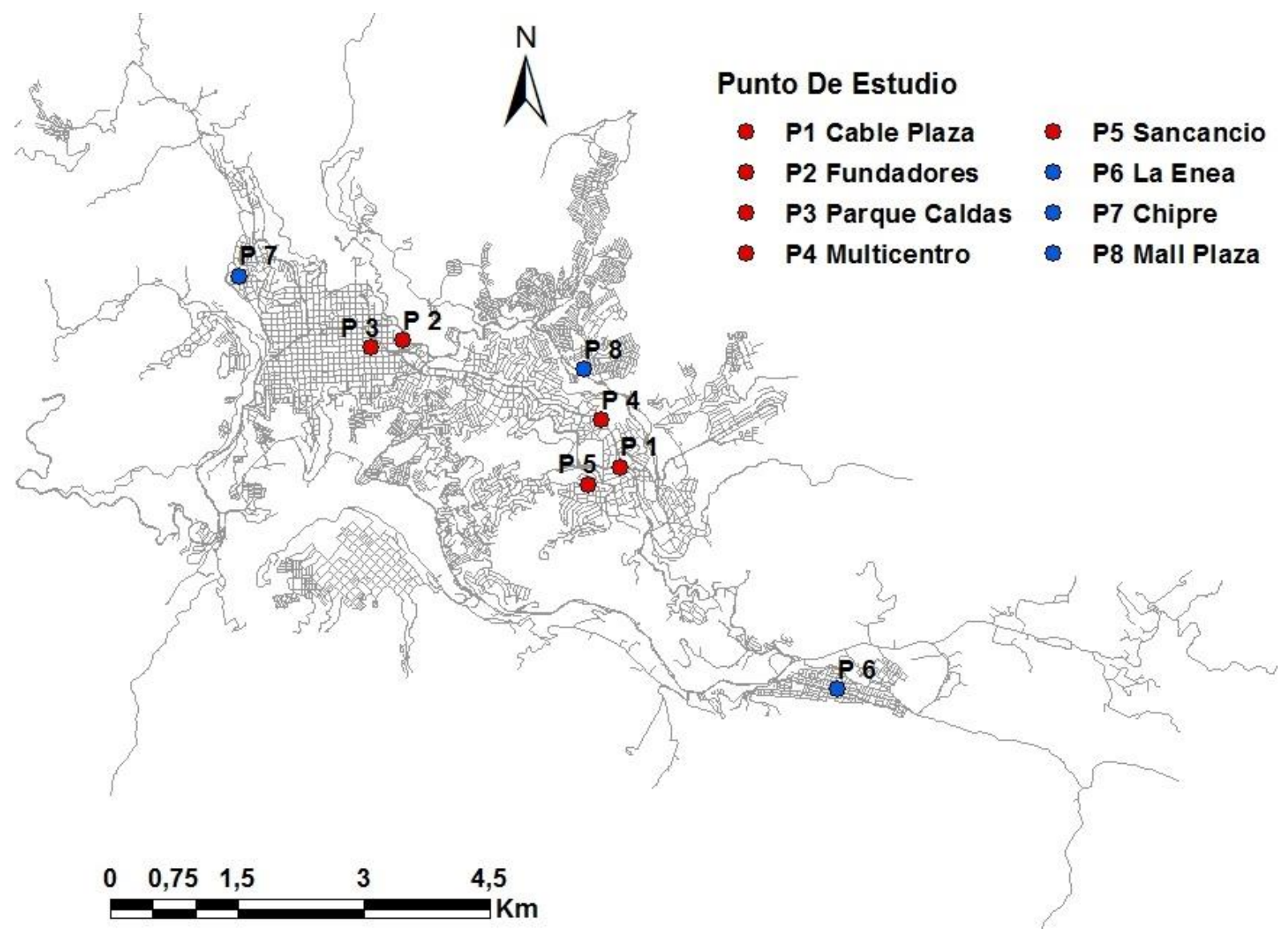

Fig. 3: Red Vial y Ubicación de nodos comerciales analizados.

La construcción de las curvas de accesibilidad se fundamenta en el algoritmo Djkstra, el cual se encuentra soportado en la extensión Network Analyst de ArcMap y permite obtener el vector de tiempo de viaje (Tvi) observado en la Exp. (1), en donde se calcula el tiempo promedio de viaje desde los incidentes (i) hacia cada una de las facilidades (j). 


$$
\overline{T_{v l}}=\frac{\sum_{j=1}^{n} t_{v i}}{(n-1)} \quad i=1,2,3, \ldots, n ; j=1,2,3, \ldots, \mathrm{n}
$$

A partir de este vector, se deriva la matriz de tiempos promedios mínimos de viaje, a la cual se le correlacionan las coordenadas geoespaciales de cada intercesión (incidente) de la red y así obtener la matriz de tiempo medios de viaje ( $\mathrm{n} \times 3$ ) con la cual se construyen las curvas isócronas de cada centro comercial. Se usó el método kriging ordinario con semivariograma lineal como modelo de predicción de valores de tiempo medio de viaje para los sectores donde no se tenían valores de entrada; con el semivariograma se identifican las propiedades de dependencia espacial entre puntos (incidentes) pertenecientes a una red analizada, obtenido mediante la Exp. (2), donde $Z_{(x)}=$ valor de la variable en un sitio con coordenadas $\mathrm{x}, \mathrm{y} ; Z_{(x+h)}=$ otro valor muestral separado por una distancia $h ; n=$ número de parejas que se encuentran separadas por dicha distancia.

$$
\overline{\gamma_{(h)}}=\frac{\sum\left(Z_{(x+h)}-Z_{(x)}\right)^{2}}{2 n}
$$

Posterior a la construcción de las curvas de accesibilidad territorial, se procede a construir las curvas gradientes de ahorro en tiempo medio de viaje, para la cual se utilizó la Exp. (3), en donde $H_{(i)}=$ porcentaje de ahorro en el nodo i; $T_{v i(a c t)}=$ tiempo de viaje actual en el nodo i; $T_{v i(f u t)}=$ tiempo de viaje futuro en el nodo i.

$$
H_{(i)}=\left[1-\left(\frac{T_{v i(f u t)}}{T_{v i(a c t)}}\right)\right] * 100 \%
$$

Etapa 4, Análisis de cobertura. Una vez elaboradas las curvas isócronas, se procede a correlacionar la información con el total de población y área de la zona de estudio, dentro de la cual se clasifica la población según el estrato socioeconómico para posteriormente ser procesadas mediante gráficas y determinar los porcentajes de cobertura hacía cada nodo o centro comercial.

\section{RESULTADOS Y DISCUSIÓN}

Como resultado del análisis de accesibilidad, fue posible la construcción de las curvas isócronas para cada sistema de nodos comerciales, Escenario Actual y Escenario Futuro, mediante lo cual se podrá observar la variación en tiempo de viaje cada cinco (5) minutos, para cada caso y para su comparación.

\section{Escenario Actual}

En la Fig. 4. se presentan los resultados obtenidos en el analisis de accesibilidad territorial del sistema de centros comerciales existentes en la ciudad, Escenario Actual, presentados en intervalos de cada 5 minutos. El menor tiempo medio de viaje hacia los nodos comeriales se encuentra sombreado en color verde y el mayor valor de tiempo medio de viaje se encuentra sombreado en color rojo. Se destaca una mejor cobertura hacia los dos polos comerciales y de servicios de la ciudad, el sector centro y el sector denominado "El cable", observándose unas características de accesibilidad limitadas para la población residente en la periferia.

El municipio contiguo de Villamaría, al sur, refiere las peores condiciones de accesibilidad hacia los nodos comerciales, siendo ésta una población de cierta capacidad adquisitiva que carece de dicho servicio. En relación con la cobertura espacial de cada estrato, en la Fig. 5(a) se observa el porcentaje de población cubierta por las curvas isócronas, encontrando que el $50 \%$ de la población perteneciente a los estratos 5 y 6 deben invertir entre 8 y 9 minutos para acceder a los centros comerciales, el resto de estratos debe invertir entre 14 y 18 minutos (Zuluaga y Escobar, 2017), es decir, hasta un 100\% más de tiempo medio de viaje. Adicionalmente, en la Fig. 5(b) se presenta la variación del porcentaje acumulado de cobertura para las variables área y población según las curvas isócronas obtenidas. Es posible observar que el $50 \%$ de la población del área de estudio debe invertir hasta 17 minutos de tiempo medio de viaje, valor que asciende a 22,5 minutos en relación con la variable área.

\section{Escenario Futuro}

En la Fig. 6 se presentan las curvas isócronas obtenidas para la el escenario futuro en la zona de estudio. De forma general, al comparar los sectores periféricos, es posible identificar una reducción en tiempo de viaje de alrededor de 10 minutos en relación al escenario actual, logrando así una mejora en la accesibilidad hacia dichos sectores. En el caso de Villamaría, no es posible obtener una mejora en las condiciones de 
accesibilidad debido a que el casco urbano del municipio posee una única conexión con la ciudad de Manizales limitando así las posibilidades de mejora.

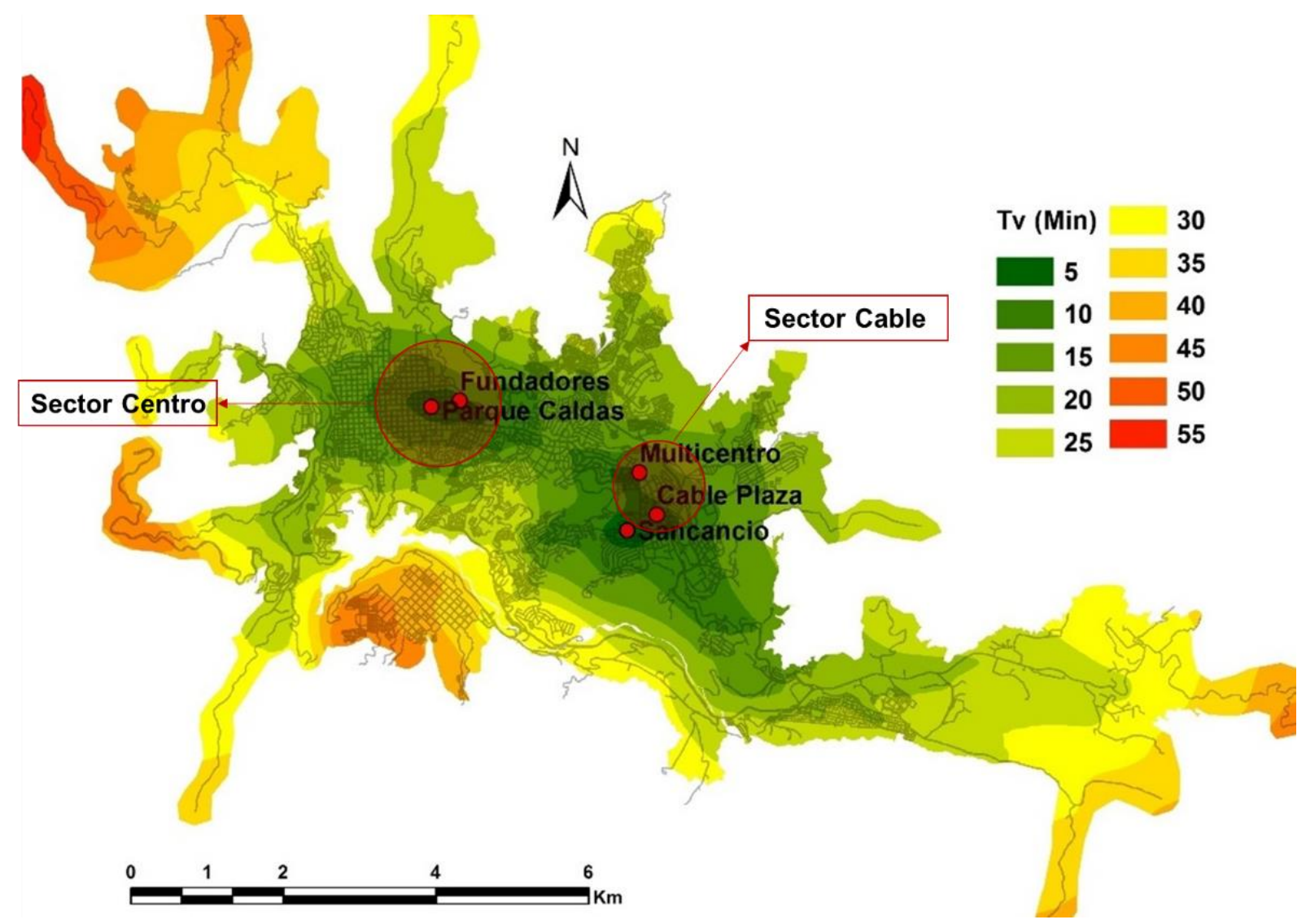

Fig. 4: Accesibilidad territorial. Escenario actual (Adaptada de Zuluaga y Escobar, 2017)
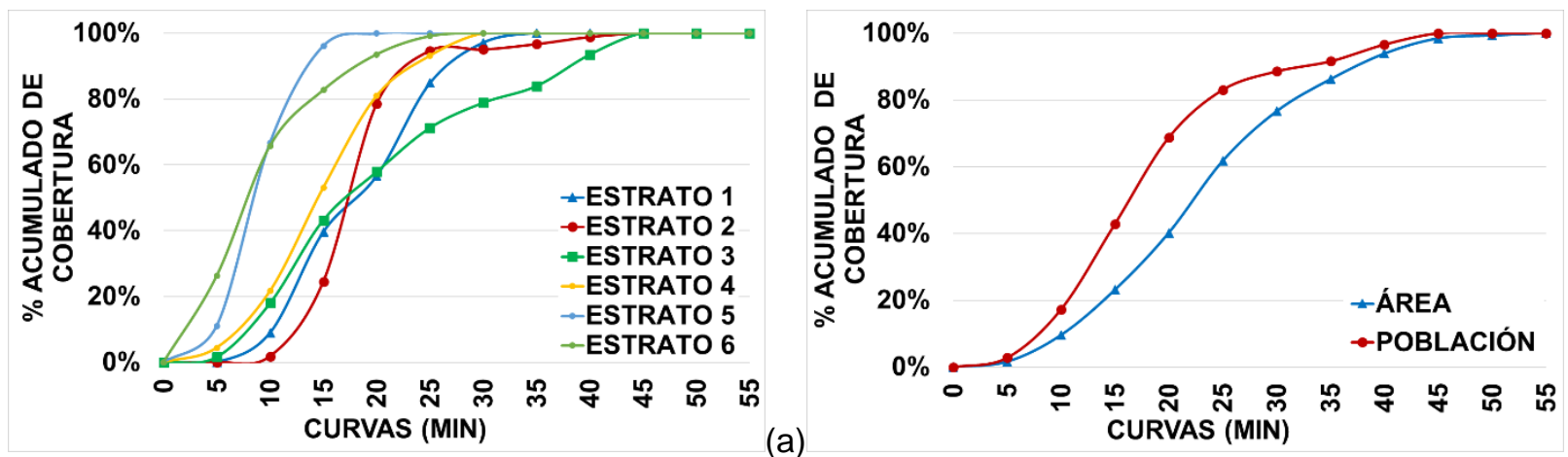

Fig. 5: (a) Porcentaje Acumulado de Cobertura Vs Tiempo Medio de Viaje según Estrato Socioeconómico en el Escenario Actual (Zuluaga y Escobar, 2017); (b) Tiempo Medio de Viaje Vs \% de Cobertura Acumulado para Población y de Área Totales en el Escenario Actual (Zuluaga y Escobar, 2017).

Por otra parte, en la Fig. 7(a), se analiza el porcentaje de cobertura acumulado según el estrato socioeconómico al cual pertenece la población, es de resaltar que la totalidad de los estratos garantizan una cobertura superior al $60 \%$ en un límite de tiempo menor a 20 minutos; a pesar que los estratos 6 y 5 siguen siendo los que refieren menores valores en tiempo de acceso, es decir, mejores condiciones de accesibilidad, se encontró que al comparar los tiempos medios de viaje para un porcentaje de cobertura poblacional del $50 \%$, por estrato, son precisamente los estratos altos los que refieren muy poca mejora de las condiciones de accesibilidad, aproximadamente $2 \%$ de disminución de tiempo medio de viaje para el estrato 6 y un aumento del 13\% en el tiempo medio de viaje para el estrato 5. Los estratos de mayor porcentaje de disminución en el tiempo medio de viaje fueron el 2 y el 4 con $27 \%$ y 19\%, respectivamente. En la Fig. 7(b) se presenta la variación del porcentaje acumulado de cobertura para las variables área y población según las curvas isócronas obtenidas, en el escenario futuro se tiene que el $50 \%$ de la población del área de estudio debe invertir hasta 13 minutos de tiempo medio de viaje, es decir, aproximadamente 4 minutos menos que en el escenario actual. Al analizar de forma más detallada, se observa que el $25 \%$ de la población disminuiría su tiempo medio de viaje para llegar a un nodo comercial en aproximadamente un $25 \%$ al pasar de tener la necesidad de invertir unos 12 minutos a invertir unos 9 minutos. 


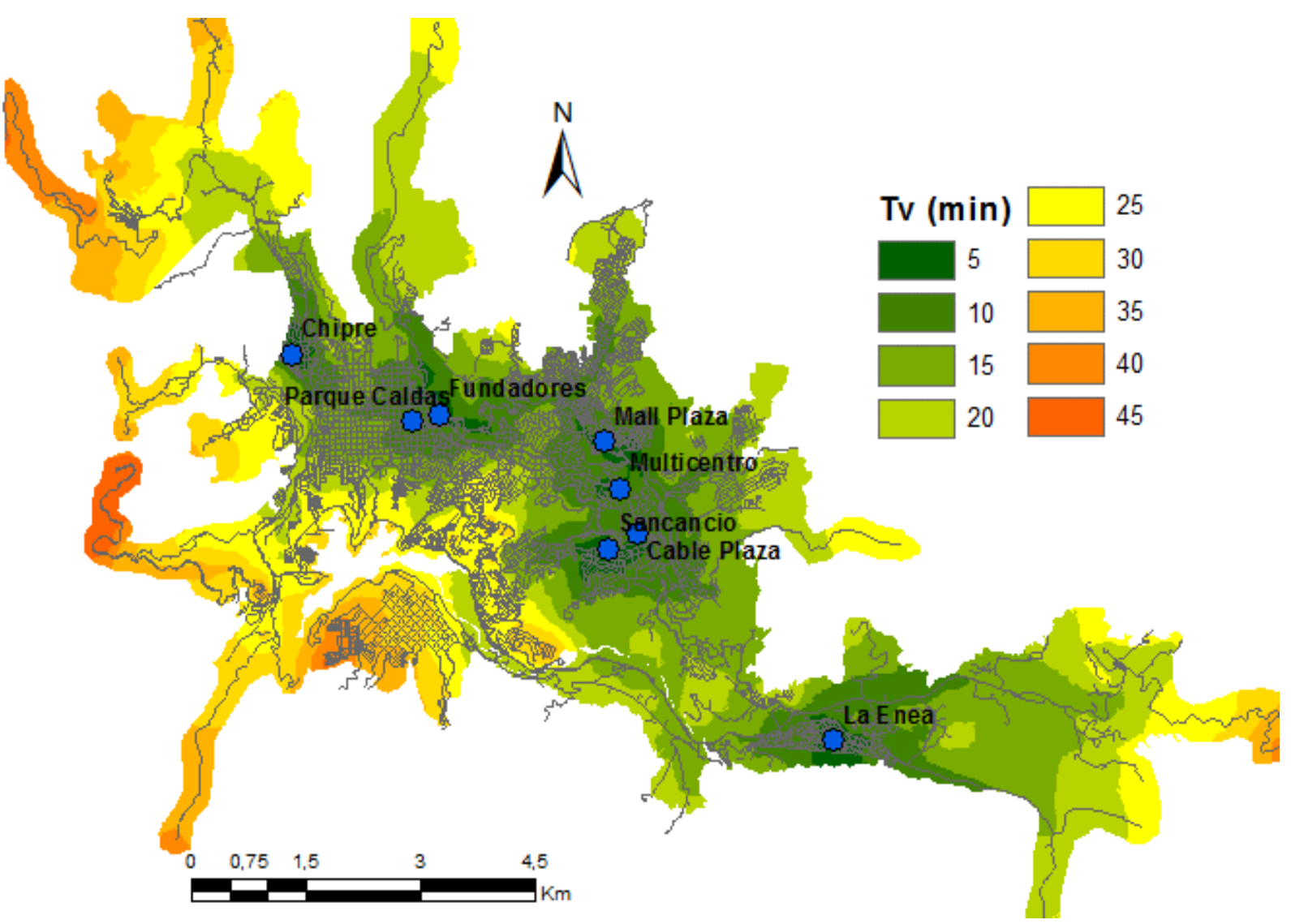

Fig. 6: Accesibilidad territorial. Escenario Futuro.
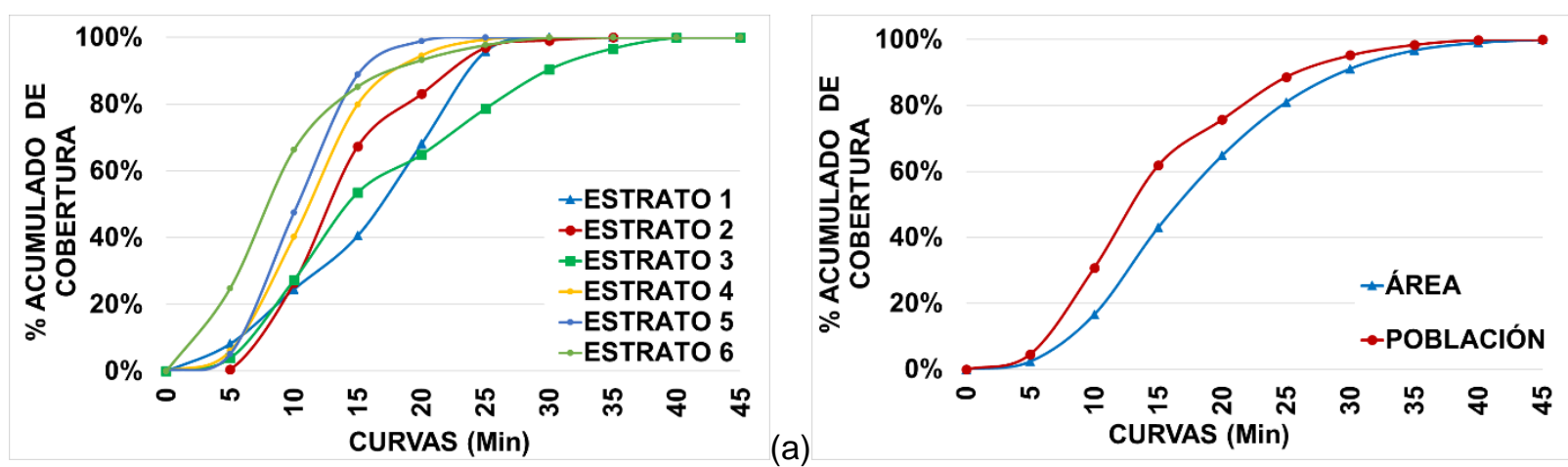

Fig. 7: (a) Porcentaje Acumulado de Cobertura Vs Tiempo Medio de Viaje según Estrato Socioeconómico en el Escenario Futuro; (b) Tiempo Medio de Viaje vs \% de Cobertura Acumulado para Población y de Área Totales en el Escenario Futuro.

\section{Gradiente entre escenarios}

En la Fig. 8 se presentan las curvas gradiente de ahorro porcentual de tiempo medio de viaje al comparar ambos escenarios estudiados; los sectores en color verde son los que refieren unos mayores porcentajes de ahorro mientras que los sectores sombreados en color rojo son aquellos que refieren los menores porcentajes de ahorro en tiempo medio de viaje. Se identifica que el sector suroriente del área de estudio (Barrio La Enea) es el que mayores beneficios reporta dada la inserción de un nodo comercial, observándose cómo el ahorro de tiempo medio de viaje se extienda hasta el municipio de Villamaría, encontrando ahorros que van del $35 \%$ al $85 \%$ en relación con el escenario actual. Así mismo, el sector del Barrio Chipre y la Linda (Zona Noroccidental del mapa), se identifican mejoras en las condiciones de accesibilidad territorial, registrándose porcentaje de ahorro de entre $30 \%$ y $45 \%$ en tiempo medio de viaje en relación con el escenario actual. Adicionalmente, se destaca el ahorro en tiempo de viaje para el sector de Mall Plaza (Norte, Comuna 5), con un valor superior al $40 \%$ de ahorro, sin embargo, se aprecia que el área afectada es menor en comparación a las dos zonas descritas anteriormente. 


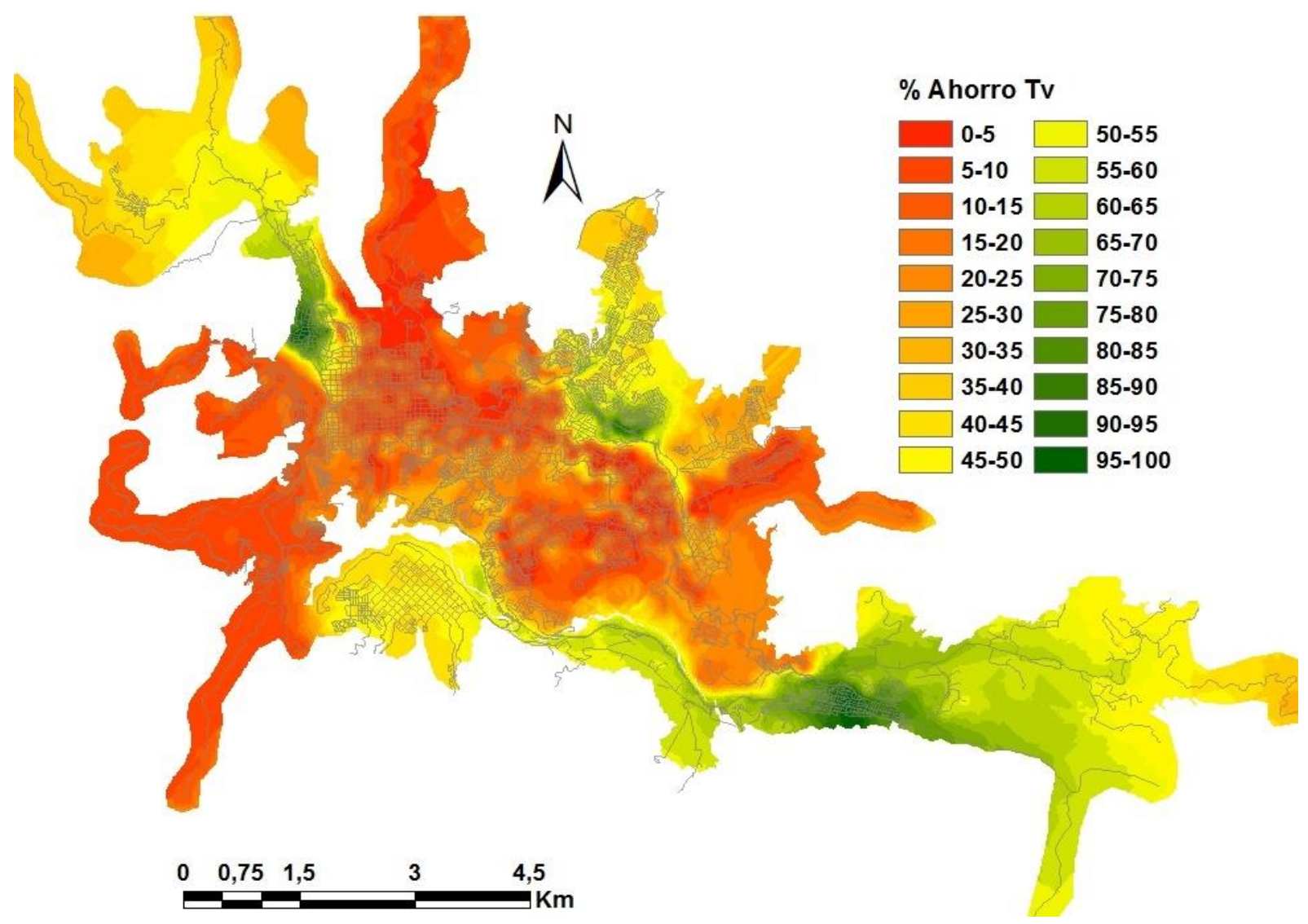

Fig. 8: Ahorro en Tiempo de Viaje entre escenarios.

En la Fig. 9(a) se presenta el porcentaje de ahorro en tiempo medio de viaje para cada estrato socioeconómico según el porcentaje acumulado de cobertura poblacional. Es posible establecer que para un $25 \%$ de cobertura poblacional, los estratos más beneficiados son el 4, 2, 3, 1, 5 y 6 con porcentaje de ahorro de tiempo medio de viaje de entre el $57 \%$ para el estrato 4 y el $22 \%$ para el estrato 6; para un $50 \%$ de cobertura poblacional, los estratos más beneficiados son el 2, 3, 4, 5, 1 y 6 con porcentaje de ahorro de tiempo medio de viaje de entre el $41 \%$ para el estrato 2 y el $13 \%$ para el estrato 6 ; así como para un $75 \%$ de cobertura poblacional, los estratos más beneficiados son el $2,3,4,5,1$ y 6 con porcentaje de ahorro de tiempo medio de viaje de entre el $18 \%$ para el estrato 2 y el $8 \%$ para el estrato 6 . Lo anterior permite establecer que en relación con el porcentaje poblacional según estrato socioeconómico, el escenario futuro beneficiará en mayor proporción al estrato 2, y en menor proporción al estrato 6. Finalmente, en la Fig. 9(b) se observa la variabilidad del porcentaje acumulado de cobertura de las variables población y área según el porcentaje de ahorro en tiempo medio de viaje.

Se encontró que el $75 \%$ de la población y del área de estudio, refieren un $12,5 \%$ de porcentaje de ahorro en tiempo medio de viaje en relación con el escenario actual; el $50 \%$ de la población refiere un $26 \%$ de ahorro mientras que el $50 \%$ de área de estudio refiere un 34\% de ahorro en tiempo medio de viaje; así mismo, el $25 \%$ de población tendría un $46 \%$ de ahorro en tiempo medio de viaje mientras que el $25 \%$ del área de estudio registrará aproximadamente un $50 \%$ de ahorro en tiempo medio de viaje.

Los valores de porcentaje de ahorro para un mismo valor de cobertura de las variables población y área (p.e. $25 \%$ y $50 \%$ ), están directamente relacionadas con las características de densidad poblacional de los diferentes sectores del área de estudio, es así que estos resultados coinciden claramente con los resultados de las curvas isócronas obtenidos, en los cuales el sector del área de estudio que refiere mayores beneficios en relación con el escenario futuro (franja sur y suroriente), son sectores donde hay una baja densidad poblacional en relación con los otros dos sectores más beneficiados (Barrio Chipre y Comuna 5 al norte).

Es de especial importancia realizar un estudio de accesibilidad detallado, con el fin de determinar el impacto en la población al momento de implementar estructuras comerciales, sin embargo, se recomienda añadir un estudio de afectación en la movilidad. Esto debido a la gran carga vehicular de camiones que podría generar en la zona, dada la construcción del nodo comercial y dada la operación futura del mismo, ya que seguramente atraerá diferentes configuraciones de vehículos de carga, lo cual abre una nueva línea de investigación relacionada con lo que se pretende definir en el presente estudio. 

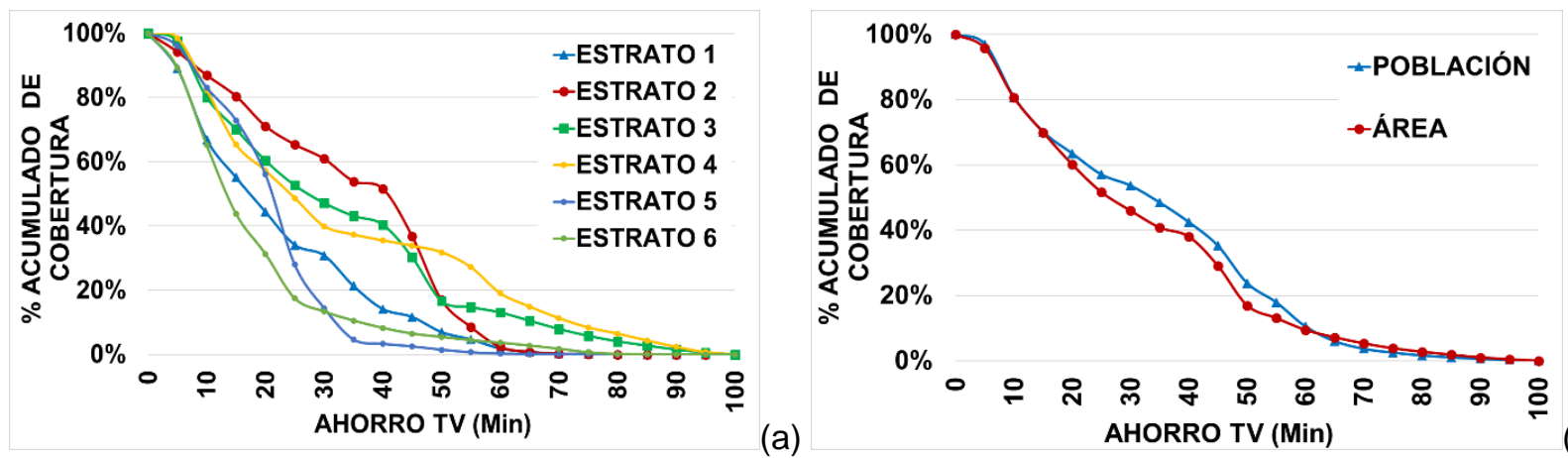

Fig. 9: (a) Porcentaje de ahorro en tiempo de viaje Vs Porcentaje de cobertura acumulado según estrato socioeconómico, gradiente entre escenarios; (b) Porcentaje de Ahorro en Tiempo de Viaje Vs Porcentaje de Cobertura Acumulado en el Total de Población y Área, gradiente entre escenarios.

\section{CONCLUSIONES}

Se concluye que la implementación de nuevos nodos o centros comerciales garantizaría una mejora en las condiciones de accesibilidad a los ciudadanos residentes en la periferia del área de estudio y a la población en general, lo cual reflejaría una disminución considerable en tiempos de viaje que garantizan una mejor calidad de vida, además de impulsar el crecimiento económico de la ciudad. Para el escenario futuro son los estratos considerados populares, es decir, los estratos 1,2 y 3 , los que se identifican como más beneficiados dada la inserción de los nuevos nodos comerciales, a pesar de que seguirían siendo los estratos altos, estratos 5 y 6 , los que continúan con las mejores condiciones de accesibilidad dada la actual red de infraestructuras del transporte y la actual y futura disposición geográfica de los nodos comerciales evaluados. Adicionalmente, se tiene que, en relación con el porcentaje poblacional según estrato socioeconómico, el escenario futuro beneficiará en mayor proporción al estrato 2 , y en menor proporción al estrato 6 , dato de suma importancia al momento de determinar la población objetivo y formular un nodo comercial acorde a la capacidad económica del sector.

\section{AGRADECIMIENTOS}

Los autores expresan su agradecimiento a los estudiantes pertenecientes al semillero de investigación en Movilidad Sostenible y al Semillero de investigación en Planificación de Urbana de la Universidad Nacional de Colombia - Sede Manizales.

\section{REFERENCIAS}

Alcaldía de Manizales, Secretaría de Tránsito y Transporte, Plan de Movilidad de la ciudad de Manizales 2010 - 2040 , Universidad Nacional de Colombia, Manizales, Colombia, (2011).

Batty, M., Accessibility: in search of a unified theory, doi:10.1068/b3602ed, Environment and Planning B: Planning and Design, 36(2), 191-194 (2009).

Bocarejo, J.P. y Oviedo, D.R., Transport accessibility and social inequities: a tool for identification of mobility needs and evaluation of transport investments, doi:10.1016/j.jtrangeo.2011.12.004, Journal of Transport Geography, 24, 142-154 (2012).

Calcuttawala, Z., Landscapes of information and consumption: A location analysis of public libraries in Calcutta, en Edward D. Garten, Delmus E. Williams, James M. Nyce (ed.), Advances in Library Administration and Organization, Emerald Group Publishing Limited, 24, 319-388 (2006).

Centro de Información y Estadística - CIE, Población Urbana 2016, (en la web: https://goo.gl/FEuiWo, acceso: 9 de junio de 2017), Alcaldía de Manizales, Secretaría de Planeación (2016).

Chardon, A.C., Reasentamiento y hábitat en zonas urbanas, una reflexión en Manizales, https://goo.gl/krMJo3, ISSN: 2027-2103, Cuadernos de vivienda y urbanismo, 1(2), 226-247 (2008).

Engwicht, D., Reclaiming Our Cities and Towns: Better Living with Less Traffic, $1^{\text {a }}$ Ed., 1-190, New Society Publishers, Philadelphia (1993).

Escobar D.A., García F.J. y Tolosa R.A., Análisis de Accesibilidad Territorial a Nivel Regional, 1a Ed., 1-157, Editorial Universidad Nacional de Colombia, Manizales, Colombia (2013).

Escobar, D.A., Cadena, C. y Salas, A., Cobertura Geoespacial de nodos de actividad primaria. Análisis de los aportes a la sostenibilidad urbana mediante un estudio de accesibilidad territorial, doi:http:/dx.doi.org/10.14508/reia.2015.12.23.1327, Revista EIA, 12(23), 13-27 (2015a).

Escobar, D.A., Tapasco, O.A. y Giraldo, J.A., Medición de Desempeño del Sistema de Transporte Cable Aéreo de la Ciudad de Manizales en Colombia, usando Tres Enfoques: Analítico, Simulado y de Accesibilidad Urbana, doi: http://dx.doi.org/10.4067/S0718-07642015000600020, Inf. Tecnol., 26(6), 199-210 (2015b). 
Garrocho, C. y Campos, J., Un indicador de accesibilidad a unidades de servicios clave para ciudades mexicanas: fundamentos, diseño y aplicación, https://goo.gl/9oq4Hp, ISSN:1405-8421, Economía, Sociedad y Territorio, 6(22), 160 (2006).

Garrocho, C., Chávez, T. y Álvarez, J.A., La Dimensión espacial de la competencia comercial, 1ª Ed., 1-200, Librería de El Colegio Mexiquense, México D.F., México (2003).

Geurs, K.T. y Ritsema van Eck, J.R., Accessibility Measures: Review and Applications. Evaluation of Accessibility Impacts of Land-use Transport Scenarios, and Related Social and Economic Impacts. (En la web: https://goo.gl/xkrKU1, acceso: 4 de Agosto de 2011), National Institute of Public Health and the Environment, Utrecht, Netherland (2001)

Geurs, K.T. y Van Wee, B., Accessibility evaluation of land-use and transport strategies: review and research directions, doi: https://doi.org/10.1016/j.jtrangeo.2003.10.005, J. of Transport Geography, 12(2), 127-140 (2004).

Gobernación de Caldas, Fondo para el Financiamiento del Sector Agropecuario, FINAGRO, Biblioteca Luis Ángel Arango del Banco de la República, Datos del Departamento de Caldas (en la web: https://goo.gl/tZc2fh, acceso: 15 de junio de 2017), Manizales, Colombia (2016).

Hansen, W.G., How accessibility shapes land use, doi: https://doi.org/10.1080/01944365908978307, Journal of the American Institute of Planners, 25 (2), 73-76 (1959).

Hasibuan, H.S., Soemardi, T.P., Koestoer, R. y Moersidik, S., The Role of Transit Oriented Development in constructing urban environment sustainability, the case of Jabodetabek, Indonesia, doi:10.1016/j.proenv.2014.03.075, Procedia Environmental Sciences, 20, 622-631 (2014).

Manizales Como Vamos - MCV, Cómo Vamos en Movilidad (en la web: https://goo.gl/B3u8XY, acceso: 9 de junio de 2017), Manizales, Colombia (2016).

Martínez, H.S., La accesibilidad regional y el efecto territorial de las estructuras de transporte. Aplicación en Castilla-La mancha, https://goo.gl/yLm3Wg, ISSN: 0212-9426, Boletín de la Asociación de Geógrafos Españoles, 59, 79-103 (2012).

Morris, J.M., Dumble, P.L. y Wigan, M.R., Accessibility indicators in transport planning, doi:https://doi.org/10.1016/01912607(79)90012-8, Transportation Research Part A: General, 13(2), 91-109 (1979).

Preston, J. y Rajé, F. Accessibility, mobility and transport-related social exclusion, doi:10.1016/j.jtrangeo.2006.05.002, Journal of Transport Geography, 15(3), 151-160 (2007).

Robledo, J.E., La Ciudad de la Colonización Antioqueña. Manizales, 1aㅡ ed., 1-242, Editorial Universidad Nacional de Colombia, Manizales, Colombia (1996).

Schürmann, C., Spiekermann, K. y Wegener, M., Accessibility indicator, SASI Deliverable D5. Report to the European Commission, Berichte aus dem Institut für Raumplanung, 39, Dortmund (1997).

Torres, C.A., Ciudad informal colombiana: Barrios construidos por la gente, $1^{\underline{a}}$ ed., Editorial Universidad Nacional de Colombia, Bogotá, Colombia (2017).

Vega, A., A. multi-modal approach to sustainable accessibility in Galway, doi: https://doi.org/10.1080/20429843.2011.9727923, Regional Insights, 2(2), 15-17 (2011).

Wachs, M. y Kumagai, T.G., Physical accessibility as a social indicator, doi:https://doi.org/10.1016/0038-0121(73)900414, Socio-Economic Planning Sciences, 7(5), 437-456 (1973).

Zuluaga, J.D. y Escobar, D.A., Geomarketing Analysis for Shopping Malls in Manizales (Colombia). Accessibility approach methodology, https://goo.gl/6nS39k, ISSN:0798-1015, Revista Espacios, 38(21), 20 (2017). 